\title{
TERMINAL PATH NUMBERS FOR CERTAIN FAMILIES OF TREES
}

\section{A. MEIR and J. W. MOON}

(Received 20 June 1980)

Communicated by W. D. Wallis

\begin{abstract}
We determine the limiting distribution of the distance from the root of a tree to any nearest endnode of the tree (other than the root) for certain families of rooted trees.
\end{abstract}

1980 Mathematics subject classification (Amer. Math. Soc.): 05 C 05.

\section{Introduction}

The terminal path number $\delta\left(T_{n}\right)$ of a rooted tree $T_{n}$ with $n$ nodes is defined as follows (for definitions not given here see Harary and Palmer (1973) or Moon (1970)): if $n=1$ then $\delta\left(T_{n}\right)=0$; otherwise, $\delta\left(T_{n}\right)$ is the distance from the root of $T_{n}$ to any nearest endnode of $T_{n}$ (other than the root if it is an endnode). If $F_{F}$ denotes some family of rooted trees, let $p(n, k)$ denote the probability that $\delta\left(T_{n}\right)>k$ where the probability is taken over the trees $T_{n}$ in $\mathscr{F}$. Grimmett (1980) has derived a recurrence relation for the numbers

$$
p_{k}=\lim _{n \rightarrow \infty} p(n, k)
$$

when $\mathcal{F}$ is the family of rooted labelled trees. His argument is based on a probabilistic representation of these trees in terms of a branching process satisfying certain conditions. Our object here is to consider this problem for a fairly general class of families of rooted trees by a direct combinatorial argument. Our main results are given in Section 3 after some preliminaries in Section 2; some numerical results are given in Section 4 for labelled trees, plane trees, and binary trees. 


\section{Preliminaries}

Let $y_{n}$ denote the number of trees $T_{n}$ in a given family $\mathcal{F}$ of rooted trees; if there are weights associated with trees in $\mathscr{F}$, then each tree is counted according to its weight in these definitions. We shall assume that $\mathscr{F}$ is a simply generated family, that is, that the generating function

$$
y=y(x)=\sum_{1}^{\infty} y_{n} x^{n}
$$

satisfies a relation of the type

$$
y=x \varphi(y)
$$

where

$$
\varphi(y)=1+c_{1} y+c_{2} y^{2}+\cdots
$$

is a power series in $y$ with non-negative coefficients. This implies that the trivial tree $T_{1}$ is in $\mathcal{F}$ and that any non-trivial tree $T_{n}$ in $\mathscr{F}$ can be constructed by joining the roots of an ordered collection of smaller trees in $\mathscr{F}$-called the branches of $T_{n}$-to a new node which serves as the root of $T_{n}$. The factor $x$ in equation (1) takes the new root node into account, and the coefficients $c_{i}$ determine weights associated with the trees in $\mathscr{F}$. For further elaboration of this last point see Meir and Moon (1978); see also Otter (1949).

If $0 \leqslant k \leqslant n-1$ let $y_{n k}$ denote the number of trees $T_{n}$ in $\mathscr{F}$ such that $\delta\left(T_{n}\right) \geqslant k$. We now give a recurrence relation for the generating functions

$$
G_{k}(x)=\sum_{n=k+1}^{\infty} y_{n k} x^{n}
$$

THEOREM 1. If $y=x \varphi(y)$, then $G_{0}(x)=y(x)$ and

$$
G_{k+1}(x)=x \varphi\left(G_{k}(x)\right)-x
$$

for $k=0,1, \ldots$

Proof. It is easy to see that $\delta\left(T_{n}\right) \geqslant k+1$ for a non-trivial rooted tree $T_{n}$ if and only if $\delta(B) \geqslant k$ for each branch $B$ of $T_{n}$. Relation (2) follows immediately from this observation and assumption (1); the term $-x$ excludes the trivial tree $T_{1}$ that has no branches.

We shall use the following results in the next section. 
LeMMA 1. Suppose

$$
\varphi(t)=1+c_{1} t+c_{2} t^{2}+\cdots
$$

is a regular function of $t$ when $|t|<R \leqslant+\infty$ and let

$$
y=y(x)=x+y_{2} x^{2}+y_{3} x^{3}+\cdots
$$

denote the solution of $y(x)=x \varphi(y(x))$ in a neighbourhood of $x=0$. If

(i) $c_{1}>0$ and $c_{j}>0$ for some $j \geqslant 2$,

(ii) $c_{i}>0$ for $i \geqslant 2$, and

(iii) $\tau \varphi^{\prime}(\tau)=\varphi(\tau)$ for some $\tau$, where $0<\tau<R$, then

$$
y_{n} \sim c \rho^{-n} n^{-3 / 2}
$$

as $n \rightarrow \infty$, where $\rho=\tau / \varphi(\tau)$ and $c=\left\{\varphi(\tau) /\left(2 \pi \varphi^{\prime \prime}(\tau)\right)\right\}^{1 / 2}$. Furthermore, if $x \varphi^{\prime}(y(x))=\Sigma_{1}^{\infty} d_{n} x^{n}$, then

$$
d_{n} \sim c \varphi^{\prime \prime}(\tau) \rho^{-n+1} n^{-3 / 2}
$$

as $n \rightarrow \infty$.

LEMMA 2. Let $A(x)=\sum_{0}^{\infty} a_{n} x^{n}, B(x)=\Sigma_{0}^{\infty} b_{n} x^{n}$, and $A(x) B(x)=\Sigma_{0}^{\infty} c_{n} x^{n}$, and suppose there exist positive constants $a$ and $\rho$ such that

$$
a_{n} \sim a \rho^{-n} n^{-1 / 2} \quad \text { and } \quad b_{n}=O\left(\rho^{-n} n^{-3 / 2}\right)
$$

as $n \rightarrow \infty$. If $B(\rho) \neq 0$, then

$$
c_{n} \sim B(\rho) a_{n}
$$

as $n \rightarrow \infty$.

Relation (3) was proved in Meir and Moon (1978) and the proof of relation (4) is essentially the same except that Darboux's theorem is applied to expansion (3.5) in that paper instead of to expansion (3.3); we remark that a result closely related to relation (3) was proved earlier in Otter (1949). Lemma 2 was proved in Meir and Moon (1977).

\section{Main results}

We now determine the limiting behaviour of the probability $p(n, k)=y_{n k} / y_{n}$ that $\delta\left(T_{n}\right) \geqslant k$ for a tree $T_{n}$ chosen from the simply generated family $\mathscr{F}$. We assume throughout this section that the function $\varphi$ that appears in relation (1) satisfies the hypothesis of Lemma 1. 
THEOREM 2. If $k$ is any fixed non-negative integer, then

$$
p_{k}=\lim _{n \rightarrow \infty} p(n, k)=\gamma_{1} \gamma_{2} \cdots \gamma_{k},
$$

where

$$
\gamma_{j}=\rho \varphi^{\prime}\left(G_{j-1}(\rho)\right)
$$

for $j>1$ and an empty product is interpreted as one.

Proof. In what follows we let $\mathcal{C}_{n}\{f(x)\}$ denote the coefficient of $x^{n}$ in any power series $f(x)$.

If we differentiate both sides of equation (2) with respect to $x$, multiply throughout by $x$, and then simplify slightly, we find that

$$
x G_{k+1}^{\prime}(x)=x \varphi^{\prime}\left(G_{k}(x)\right) \cdot x G_{k}^{\prime}(x)+G_{k+1}(x)
$$

for $k>0$. We may assume as our induction hypothesis that

$$
\mathcal{C}_{n}\left\{x G_{k}^{\prime}(x)\right\}=n y_{n k}=n p(n, k) y_{n} \sim c p_{k} \rho^{-n} n^{-1 / 2}
$$

as $n \rightarrow \infty$, in view of relation (3). Furthermore, it follows from relation (4) that

$$
\mathcal{C}_{n}\left\{x \varphi^{\prime}\left(G_{k}(x)\right)\right\} \leqslant \mathcal{C}_{n}\left\{x \varphi^{\prime}(y)\right\}=O\left(\rho^{-n} n^{-3 / 2}\right) .
$$

Therefore, since

$$
\mathcal{C}_{n}\left\{G_{k+1}(x)\right\} \leqslant y_{n}=O\left(\rho^{-n} n^{-3 / 2}\right),
$$

it follows from (5) and Lemma 2 that

$$
n p(n, k+1) y_{n}=\mathcal{C}_{n}\left\{x G_{k+1}^{\prime}(x)\right\} \sim \rho \varphi^{\prime}\left(G_{k}(\rho)\right) \cdot n p(n, k) y_{n},
$$

or that

$$
p(n, k+1) \rightarrow \gamma_{k+1} p_{k}=p_{k+1}
$$

as $n \rightarrow \infty$. This suffices to complete the proof of the theorem.

COROLlary 2.1. There exists a constant $A$ that depends on $\mathscr{F}$ such that

$$
p_{k}\left(\rho c_{1}\right)^{-k} \rightarrow A
$$

as $k \rightarrow \infty$.

Proof. Let $\beta_{k}=G_{k}(\rho)$ for $k=0,1, \ldots$ Then $\beta_{0}=y(\rho)=\tau$ by Lemma 1 , and

$$
\beta_{k+1}=g\left(\beta_{k}\right)
$$

where

$$
g(t)=\rho(\varphi(t)-1)
$$

in view of Theorem 1 . If $0<t \leqslant \tau$ then

$$
g(t)=\rho\left(c_{1} t+c_{2} t^{2}+\cdots\right)=t \rho\left(c_{1}+c_{2} t+\cdots\right)<t \rho \varphi^{\prime}(\tau)=t
$$


since $c_{i}>0$ for some $i \geqslant 2$ and since $\rho \varphi^{\prime}(\tau)=1$ by Lemma 1. It follows, therefore, that the sequence $\beta_{0}, \beta_{1}, \ldots$ decreases monotonically to a limit which must necessarily be zero. This implies, since $0<\rho c_{1}<1$, that

$$
\beta_{k}\left(\rho c_{1}\right)^{-k} \rightarrow a
$$

for some constant $a$ as $k \rightarrow \infty$; see DeBruijn (1970), Section 8.3.

Now

$$
\gamma_{j}=\rho \varphi^{\prime}\left(\beta_{j-1}\right)=\rho c_{1}\left(1+O\left(\beta_{j-1}\right)\right)=\rho c_{1}\left(1+O\left(\left(c_{1} \rho\right)^{j-1}\right)\right)
$$

since $\varphi^{\prime}(t)$ is regular when $|t|<R$ and $\beta_{j-1} \leqslant \tau<R$ when $j \geqslant 1$. Consequently,

$$
p_{k}\left(\rho c_{1}\right)^{-k}=\prod_{1}^{k}\left(\gamma_{j} / \rho c_{1}\right)=\prod_{1}^{k}\left(1+O\left(\left(c_{1} \rho\right)^{j-1}\right)\right) \rightarrow A
$$

for some constant $A$ as $k \rightarrow \infty$, since $\Sigma\left(c_{1} \rho\right)^{j-1}$ converges. This proves the required result.

Let $\mu(n)$ denote the expected value of $\delta\left(T_{n}\right)$ over all trees $T_{n}$ in $\mathscr{F}$. Before determining the limiting value of $\mu(n)$ we need to introduce some more terminology and results.

If the tree $T_{n}$ is rooted at node $r$ suppose we select an edge $r s$ incident with node $r$; we next select an edge $s u$ incident with node $s$ where $u \neq r$, and so on. Let $c\left(T_{n}\right)$ denote the number of edges selected before reaching an endnode of $T_{n}$ (other than $r$ if $r$ is an endnode) where the process terminates. We assume that at each step the next edge chosen is chosen at random from the admissible edges; we define $c\left(T_{n}\right)$ to be zero when $n=1$. If $0<k \leqslant n-1$, let $q(n, k)$ denote the probability that $c\left(T_{n}\right)>k$ where the probability is taken over all trees $T_{n}$ in $\mathscr{F}$. It follows from results in Meir and Moon (1975) that

$$
q_{k}=\lim _{n \rightarrow \infty} q(n, k)=(1-\rho / \tau)^{k-1}(1+(k-1) \rho / \tau)
$$

for each non-negative integer $k$, and that

$$
\lim _{n \rightarrow \infty} \sum_{k=1}^{n-1} q(n, k)=\sum_{k=1}^{\infty} q_{k}=2 \tau / \rho-1 .
$$

Corollary 2.2. $\mu(n) \rightarrow p_{1}+p_{2}+\cdots$ as $n \rightarrow \infty$.

Proof. If $\delta\left(T_{n}\right) \geqslant k$, then it must be that $c\left(T_{n}\right) \geqslant k$. It follows readily from this observation that

$$
p(n, k)<q(n, k)
$$

for all $n$ and $k$. 
Let the functions $P_{n}(t), P(t), Q_{n}(t)$ and $Q(t)$, where $n=1,2, \ldots$ and $t$ is non-negative real variable, be defined by the relations $P_{n}(t)=p(n,[t+1])$, $P(t)=p_{[t+1]}, Q_{n}(t)=q(n,[t+1])$, and $Q(t)=q_{[t+1]}$. In particular,

$$
P_{n}(t)=Q_{n}(t)=0
$$

if $t \geqslant n-1$. It follows from Corollary 2.1 and (6) that

$$
P_{n}(t) \rightarrow P(t) \quad \text { and } \quad Q_{n}(t) \rightarrow Q(t)
$$

for each fixed value of $t$ as $n \rightarrow \infty$. Furthermore,

$$
P_{n}(t) \leqslant Q_{n}(t)
$$

for all $n$ and $t$, by (8); and

$$
\int Q_{n}(t) \rightarrow \int Q(t)
$$

as $n \rightarrow \infty$, by (7), where the integrals are over the interval $[0, \infty)$.

We may apply Fatou's Lemma (see Royden (1968), p. 226) to the functions $Q_{n}(t)-P_{n}(t)$ and conclude that

$$
\begin{aligned}
\int(Q(t)-P(t)) & \leqslant \liminf _{n \rightarrow \infty} \int\left(Q_{n}(t)-P_{n}(t)\right) \\
& \leqslant \limsup _{n \rightarrow \infty} \int Q_{n}(t)-\limsup _{n \rightarrow \infty} \int P_{n}(t) \\
& =\int Q(t)-\limsup _{n \rightarrow \infty} \int P_{n}(t)
\end{aligned}
$$

whence,

$$
\limsup _{n \rightarrow \infty} \int P_{n}(t) \leqslant \int P(t)
$$

But if we apply Fatou's Lemma to the functions $P_{n}(t)$ we find that

$$
\liminf _{n \rightarrow \infty} \int P_{n}(t) \geqslant \int P(t) .
$$

It follows, therefore, that

$$
\mu(n)=\int P_{n}(t) \rightarrow \int P(t)=p_{1}+p_{2}+\cdots
$$

as $n \rightarrow \infty$, as required.

\section{Results for particular cases}

We now consider the limiting values of $p(n, k)$, for fixed $k$, for the following families $\mathcal{F}$ of simply generated trees: labelled trees for which $c_{i}=1 / i$ ! for all $i$, 
plane trees for which $c_{i}=1$ for all $i$, and binary trees for which $c_{2}=1$ and $c_{i}=0$ otherwise (see Meir and Moon (1978)).

THEOREM 3. If $\mathcal{F}$ denotes the family of rooted labelled trees, then

$$
p_{k}=e^{-k+\beta_{0}+\beta_{1}+\cdots+\beta_{k-1}}
$$

where $\beta_{0}=1$ and

$$
\beta_{k+1}=e^{\beta_{k}-1}-e^{-1}
$$

for $k=0,1, \ldots$

Proof. In this case

$$
y=x e^{y}=\sum_{1}^{\infty} n^{n-1} \frac{x^{n}}{n !}
$$

and $\varphi(t)=e^{t}$, so $\tau=1$ and $\rho=e^{-1}$. The recurrence relation for the numbers $\beta_{k}=G_{k}\left(e^{-1}\right)$ follows from Theorem 1 ; and the formula for $p_{k}$ follows from Theorem 2 since

$$
\gamma_{k+1}=\rho \varphi^{\prime}\left(G_{k}(\rho)\right)=e^{\beta_{k}-1} .
$$

If we let $\alpha_{k}=\beta_{k}-1$ then $\alpha_{0}=0$,

$$
\alpha_{k+1}=\beta_{k+1}-1=e^{\beta_{k}}-e^{-1}-1=e^{\alpha_{k}-1}-e^{-1}-1 \text {, }
$$

and

$$
p_{k}=e^{\alpha_{0}+\alpha_{1}+\cdots+\alpha_{k-1}} \text {. }
$$

This expression for $p_{k}$ was derived by Grimmett (1980) by a different argument.

Since $p_{k}=\gamma_{1} \gamma_{2} \cdots \gamma_{k}$ it is perhaps more convenient to work directly with the numbers

$$
\gamma_{k+1}=\rho \varphi^{\prime}\left(G_{k}(\rho)\right)=e^{\beta_{k}-1}=\beta_{k+1}+e^{-1} .
$$

Then $\gamma_{1}=1$ and

$$
\gamma_{k+1}=\exp \left(\beta_{k}-1\right)=\exp \left(\gamma_{k}-e^{-1}-1\right)=\sigma e^{\gamma_{k}}
$$

where $\sigma=\exp \left(-e^{-1}-1\right)$ for $k \geqslant 1$. The numerical values of some of these numbers, truncated after the last digit displayed, are given in Table 1.

TABLE 1.

Data for Labelled Trees

$\begin{array}{ccccccc}\mathbf{k} & \beta_{\mathbf{k}} & \beta_{\mathbf{k}} \mathrm{e}^{\mathrm{k}} & \gamma_{\mathrm{k}} & \mathrm{p}_{\mathbf{k}} & \mathrm{p}_{\mathbf{k}} \mathrm{e}^{\mathrm{k}} & \Sigma_{1}^{\mathrm{k}} \mathrm{p}_{\mathbf{i}} \\ 1 & .63212 & 1.71828 & 1.00000 & 1.00000 & 2.71828 & 1.00000 \\ 2 & .32432 & 2.39642 & .69220 & .69220 & 5.11470 & 1.69220 \\ 3 & .14093 & 2.83068 & .50881 & .35219 & 7.07411 & 2.04439 \\ 4 & .05567 & 3.03986 & .42355 & .14917 & 8.14474 & 2.19357 \\ 5 & .02106 & 3.12607 & .38894 & .05802 & 8.61108 & 2.25159 \\ 10 & .00014 & 3.17854 & .36802 & .00040 & 8.90216 & 2.28596 \\ 15 & 9 \times 10^{-7} & 3.17890 & .36788 & 2 \times 10^{-6} & 8.90418 & 2.28619 \\ 20 & 6 \times 10^{-9} & 3.17890 & .36787 & 1 \times 10^{-8} & 8.90419 & 2.28619\end{array}$


THEOREM 4. If $\mathscr{F}$ denotes the family of plane trees, then

$$
p_{k}=9 \cdot 4^{-k}\left(1+2 \cdot 4^{-k}\right)^{-2}
$$

for $k=0,1, \ldots$

Proof. In this case

$$
y=x(1-y)^{-1}=\sum_{1}^{\infty}\left(\begin{array}{c}
2 n-2 \\
n-1
\end{array}\right) \frac{x^{n}}{n}
$$

and $\varphi(t)=(1-t)^{-1}$, so $\tau=\frac{1}{2}$ and $\rho=\frac{1}{4}$. If $\beta_{k}=G_{k}\left(\frac{1}{4}\right)$, then $\beta_{0}=\frac{1}{2}$ and

$$
\beta_{k}=\frac{1}{4} \beta_{k}\left(1-\beta_{k}\right)^{-1}
$$

for $k=0,1, \ldots$, by Theorem 1 , from which it follows readily by induction that

$$
\beta_{k}=\frac{3}{2} 4^{-k}\left(1+2 \cdot 4^{-k}\right)^{-1}
$$

Now

$$
\gamma_{k+1}=\rho \varphi^{\prime}\left(G_{k}(\rho)\right)=\frac{1}{4}\left(1-\beta_{k}\right)^{-2}=\left(2 \beta_{k+1} / \beta_{k}\right)^{2}
$$

whence

$$
p_{k}=\gamma_{1} \gamma_{2} \cdots \gamma_{k}=4^{k+1} \beta_{k}^{2}=9 \cdot 4^{-k}\left(1+2 \cdot 4^{-k}\right)^{-2}
$$

as required.

It follows from Corollary 2.2 and Theorem 4 that

$$
\mu(n) \rightarrow \sum_{1}^{\infty} p_{k}=1.62297 \ldots,
$$

as $n \rightarrow \infty$, for the family of plane trees.

We adopt the notational convention that $(t)_{0}=1$ and $(t)_{j}=t(t-1) \cdots$ $(t-j+1)$ for $j=1,2, \ldots$.

THEOREM 5. If $\mathcal{F}$ denotes the family of binary trees, then

$$
p(2 n+1, k)=2^{k}(n)_{2^{k}-1} /(2 n)_{2^{k}-1} \text {. }
$$

Proof. In this case $y=x\left(1+y^{2}\right)$, whence

$$
G_{k}(x)=x^{-1}(x y)^{2^{k}}
$$

by Theorem 1 . Now

$$
y^{m}=\sum_{n=0}^{\infty} \frac{m}{n+m}\left(\begin{array}{c}
2 n+m-1 \\
n
\end{array}\right) x^{2 n+m}
$$


for $m=1,2, \ldots$, by Lagrange's inversion formula. We find, therefore, that

$$
G_{k}(x)=\sum_{n=2^{k}-1}^{\infty} \frac{2^{k}}{n+1}\left(2 n+\underset{n}{n}-2^{k}\right) x^{2 n+1}
$$

for $k=0,1, \ldots$ Consequently,

$$
p(2 n+1, k)=y_{2 n+1, k} / y_{2 n+1}=2^{k}\left(2 n+1-2^{k}\right) /\left(\begin{array}{c}
2 n \\
n
\end{array}\right)
$$

and this reduces to the expression given above.

It follows readily from Theorem 5 that

$$
\lim _{n \rightarrow \infty} p(2 n+1, k)=2^{k+1-2^{k}}
$$

for each fixed integer $k$; hence

$$
\lim _{n \rightarrow \infty} \mu(2 n+1)=\sum_{1}^{\infty} 2^{k+1-2^{k}}=1.56298 \ldots,
$$

by Tannery's theorem (see Bromwich (1931), p. 136).

\section{Acknowledgements}

We are indebted to Mr. W. Aiello for performing some numerical calculations for us. The preparation of this paper was assisted by grants from the Natural Sciences and Engineering Research Council of Canada.

\section{References}

T. Bromwich (1931), An introduction to the theory of infinite series (Macmillan, London).

N. G. De Bruijn (1970), Asymptotic methods in analysis (North-Holland, Amsterdam).

G. R. Grimmett (1980), 'Random labelled trees and their branching networks', J. Austral. Math. Soc. Ser. A 30, 229-237.

F. Harary and E. Palmer (1973), Graphical enumeration (Academic Press, New York).

A. Meir and J. W. Moon (1973), 'Climbing certain types of rooted trees, I.', Proc. Fifth British Comb. Conf., University of Aberdeen, edited by C. St. J. A. Nash-Williams and J. Sheehan, pp. 461-469. (Utilitas Mathematica Publishing Inc., Winnipeg).

A. Meir and J. W. Moon (1977), 'Packing and covering constants for certain families of trees, I', J. Graph Theory 1, 157-174.

A. Meir and J. W. Moon (1978), 'On the altitude of nodes in random trees', Canad. J. Math. 30, 997-1015.

J. W. Moon (1970), Counting labelled trees (Canadian Mathematical Congress, Montreal). 
R. Otter (1949), 'The multiplicative process', Ann. Math. Statist. 20, 206-224.

H. L. Royden (1968), Real analysis (Macmillan, New York).

\section{Mathematics Department}

University of Alberta

Edmonton, Alberta

Canada T6G 2G1 